Revista de Sociología 31(2020)55-80

DOI: https://doi.org/10.15381/rsoc.v0i31.19276

ISSN impreso: 1605-8933 / ISSN en línea: 1609-7580

\title{
Desastres: una mirada histórica y social para la gestión del riesgo
}

Sección ESTUDI0S

RECIBIDO: $15 / 10 / 2020$

APROBADO: $15 / 11 / 2020$

PUBLICADO ONLINE: 18/12/2020

\author{
Pedro Ferradas Mannucci ${ }^{1}$ \\ Ministerio de Educación del Perú \\ pferradas2015@gmail.com \\ https://orcid.org/0000-0002-7540-6211
}

\section{RESUMEN}

El artículo es una aproximación a la dimensión histórico social de los riesgos y desastres a lo largo de nuestra historia republicana. Analiza, a partir de 23 casos relevantes ocurridos en cinco periodos de la historia, como las dinámicas sociales, demográficas, económicas y políticas incidieron en tales desastres y como determinaron cambios económicos, políticos, sociales e institucionales; concluye con algunas reflexiones finales que incluyen los cambios en enfoques y estrategias frente a los desastres y los retos actuales. Destaca como en el siglo XIX fueron determinantes la precariedad económica, las crisis políticas, y la débil presencia del Estado; en las tres primeras décadas del siglo XX las estrategias para afrontar las epidemias y desde la década de 1940 el mayor impacto de los desastres sobre las ciudades en crecimiento así como las reconstrucciones propiciadas desde el Estado; en el periodo1970-2010 se relevan los cambios y los contextos que originan e inciden en el desarrollo del Sistema Nacional de Defensa Civil; y desde el año 2011, aquellos que dieron origen al Sistema Nacional de Gestión de riesgo en formación y sus retos actuales. PALABRAS CLAVE: riesgos; desastres; historia; sociedad; gestión.

\section{Disasters: a historical and social view for risk management}

\section{ABSTRACT}

The article is an approximation to the social historical dimension of risks and disasters throughout our Republican History. It analyzes, based on 23 relevant cases that occurred in five periods of history, how the social, demographic, economic and political dynamics influenced such disasters and how they determined economic, political, social and institutional changes; It concludes with some final reflections that include changes in approaches and strategies in the face of disasters and current challenges. It stands out how in the 19th century the economic precariousness, political crises, and the weak presence of the State were decisive; in the first three decades of the 20th century, the strategies to face epidemics and, since the 1940s, the greatest impact of disasters on growing cities, as well as the reconstructions promoted by the State; In the period 1970-2010, the changes and contexts that originate and affect the development of the National Civil Defense System are surveyed; and since 2011, those that gave rise to the National Risk Management System in training and its current challenges. KEYWORDS: risks; disasters; history; society; management.

1 Consultor, especialista senior de la Oficina de Defensa Nacional y Gestión de riesgo de desastres del Ministerio de Educación. Asesor de la Red ambiental peruana. 
ste trabajo tiene como propósito aproximarnos a la dimensión histórico social de los desastres en el Perú y sus causas: los riesgos que se generan en los procesos de desarrollo y las políticas públicas y prácticas en torno a ello. A diferencia de la visión tradicional centrada en los fenómenos naturales, se trata de analizar cómo las dinámicas económicas, sociales y políticas van determinando condiciones de riesgo mediante la ocupación del territorio, el incremento y concentración de la población, el deterioro de los ecosistemas, el limitado acceso a la vivienda, medios de vida seguros y las respuestas diferentes frente a los desastres (Fernández, 2007: 280-281). En contraste existen también, prácticas que han contribuido a la reducción de las condiciones de riesgo, entre las que destacan: la organización social, la reforestación, y el uso tecnologías y conocimientos ancestrales que permiten reducir los peligros de deslizamiento e inundación o los efectos de las sequías y heladas en las familias (Valcárcel, 1981: 77).

En este artículo, partimos de una aproximación a los temas mencionados, a lo largo de la historia republicana, para concluir con algunas reflexiones finales que incluyen los cambios en enfoques y estrategias frente a los desastres y los retos actuales.

\section{Desastres y epidemias en el siglo XIX}

En los primeros años de la República la capacidad de responder a los desastres, que usualmente venían acompañados de epidemias, dependía de las voluntades y capacidades de los poderes locales. Los progresos de la medicina y de los sistemas de comunicación, demoraban más años en llegar, conforme las regiones eran más pobres y apartadas. La debilidad del Estado se prolongó hasta varias décadas después de la guerra del Pacífico.

Para el Estado, la preocupación principal por los fenómenos climáticos extremos era que causaran la disminución de los productos de exportación como fue el caso de la producción del guano ante el incremento de la temperatura del mar (Fagan, Glantz,1996: 23; 1997 b: 7; IGP, 2014: 56; Franco, 2000: 133)² y la afectación de los puertos y almacenes. En segundo lugar, prestaban atención a la agricultura en las haciendas, que podían ser afectadas por la sequía o las inundaciones y por la destrucción de las vías de comunicación que disminuían el comercio.

2 Fagan destaca que El Niño y la sobre explotación fueron la causa de la crisis del Guano. Glantz advierte que el interés por El Niño adquiere relevancia en la escena internacional y en la administración política en el Perú sólo a mediados del siglo XIX cuando se perciben sus efectos adversos sobre las aves guaneras y sobre la producción del guano. Ello a pesar de que la crisis en la producción del Guano se debió en 1860 principalmente a la sobreproducción como bien señala Bonilla. 
En tercer lugar estaba, la afectación de los servicios públicos en las ciudades, las viviendas y la salud de las personas.

Los desastres desencadenados por los terremotos o las alteraciones climáticas en una sociedad caracterizada por el abuso y la discriminación (Valcárcel, 1915; Remy, 2013; Contreras, 1988), tenían más impacto dada la débil economía del país.

La lenta y deficiente atención de las emergencias en el caso de los terremotos en las ciudades y la reconstrucción de sólo parte de los bienes públicos erosionaban grandemente los recursos estatales agravando los déficits y crisis preexistentes 0 en proceso como fue el caso del terremoto y tsunami de 1868 que analizamos más adelante. Agravaban, asimismo, las frecuentes crisis políticas preexistentes (Ruiz y Castro, 2000).

Las diversas epidemias causaron graves estragos, lo que se evidenció en la disminución de la población por fallecimientos o por migración. En 1854, en la ciudad del Cusco ocurrió la epidemia de tifoidea y hepatitis; no existían ni agua ni desagüe, y menos, limpieza pública, la basura se echaba en las calles y acequias abiertas. Allí fallecieron 12,000 habitantes, el $30 \%$ del total. Las pérdidas globales entre los campesinos se estimaron en un cuarto de su población (Macera, 1977, T IV: 196).

En 1868 ocurrió el gran desastre desencadenado por un terremoto y tsunami en el sur; las ciudades más afectadas por la destrucción ${ }^{3}$ fueron: Arequipa, Moquegua, Tacna, Islay, Arica e Iquique; y el tsunami arrasó las costas entre Pisco e lquique (Giesecke y Silgado, 1981:31). Al siniestro siguieron el pillaje, el desabastecimiento y la especulación con los alimentos.

Lo ocurrido en Arica fue narrado por diferentes testigos quienes destacan que, no bien se había visto que el mar se retiraba de la costa, parte de la población corrió cerro arriba: "Muchos escucharon y escalaron o sortearon los escombros para ponerse a salvo en los últimos minutos. Muchos ya estaban en buen recaudo cuando media hora después del gran sismo un estruendo lejano se aproximó como un muro gigante y al llegar a tierra se rompió con furia" (Fernández Canque, 2007: 82).

En Arequipa, después de haberse sentido el primer impacto, todos dejaron sus casas y corrieron hacia lugares abiertos, así solo 10 personas de sus 26000 habitantes fallecieron. Pero debió esperarse casi una semana para tener en Lima noticias, dada la limitada comunicación que existía; algunos diarios titularon entonces que Arequipa "ya no existe" y que ninguna iglesia o casa se había mantenido en pie.

3 Las viviendas de entonces eran predominantemente de adobe y quincha. 
El gobierno envió provisiones y dinero a bordo del navío de guerra peruano "Unión" que debido a sus deficiencias tuvo que regresar al Callao antes de alcanzar su destino y ser reemplazado por la "Independencia", que partió sin pérdida de tiempo. La ayuda de emergencia, llegó tres semanas después del sismo a Arica.

Los salitreros del sur debieron emprender la reconstrucción de las oficinas y campamentos, así como los puertos y los almacenes gravemente dañados. El comercio de exportación de lanas de alpaca y llamas se redujo a casi la mitad como consecuencia de la destrucción de caminos y muelles (Núñez Carvallo,1997: 12).

Pero este desastre también fue una oportunidad para enriquecerse. Juan Gildemeister, dueño de una gran salitrera en Iquique fue arruinado temporalmente por la destrucción de sus propiedades. Sin embargo, aprovechando que no existía cable entre la costa pacífica y Nueva York, "apresuradamente despachó a uno de sus empleados a fin de comprar una gran cantidad de nitrato al precio normal" antes de que su precio se fuera por las nubes. Años más tarde, comenzó a comprar propiedades en el valle de Chicama y llegó a poseer 300 mil hectáreas en la hacienda Casagrande que tenía una extensión equivalente a la de Bélgica (Núñez Carvallo, 1,997: 11).

Después del terremoto de 1868, la crisis económica y sus efectos se dejaron sentir durante la mayor parte de la década de 1870 debido al colapso económico de grandes regiones de la sierra a causa de las epidemias ( Cusco, Ayacucho, Huánuco) y la carestía en el sur por los desastres y sublevaciones de 1868-1869 (Macera, 1977, tomo IV: 240)

Es así como el diario El Comercio editorializaba: "Va a terminar el malhadado 77 dejando un reguero de penurias y sufrimientos, que tienen visos de prolongarse indefinidamente (...) la ganadería en decadencia, los empleados muriéndose de hambre y un malestar en todas las clases sociales son los caracteres bien acentuados del año que va a concluir"(El Comercio, 2 enero 1878).

Meses después, se da inicio al fenómeno El Niño de 1877-1878 que ha sido considerado como el más intenso del siglo XIX y se manifestó en el Perú con grandes sequías e inundaciones. En algunas provincias del Cusco se dio la sequía descrita como "la total suspensión de aguas, hasta el extremo de secarse las fuentes y los manantiales y dejar los ríos medios secos." (Huertas, 2009: 208). Las inundaciones en el Norte hicieron que en ciudades como Lambayeque tuvieran que usar botes para transportarse y que se generara escasez en los artículos de primera necesidad dada la destrucción de los cultivos (El Comercio, 12 de marzo de 1878). 


\section{Desastres, expansión económica y crecimiento urbano 1900-1969}

Ernesto Yepes se refiere al período 1890-1930 como el de inicios de la expansión mercantil capitalista (Mejía Baca, tomo II: 302). Advierte que recién hacia fines de la primera década del siglo XX, el Perú logra recuperar los niveles de exportación previos a la guerra del Pacífico, como fue el caso del algodón y la caña de azúcar.

Entre 1876 y 1940 la población de Lima-Callao pasó de 129 mil a 595 mil habitantes; se multiplicó por 4,6 veces, mientras que la población total del país lo hizo sólo por 2,3. Otras ocho ciudades, también crecieron a mayor velocidad (Hunt, 1980: 85 y 105). Si consideramos como centros urbanos una población mayor de 20 mil habitantes, entre 1876 y 1940, el número de ciudades que superaban esta cifra pasó de tres (Lima, Callao y Arequipa) a nueve (se incorporaron Cusco, Trujillo, Chiclayo, Iquitos, Huancayo e Ica) (Contreras, 1994: 61). Como se puede observar Tumbes y Piura, escenarios relevantes en las inundaciones por el Fenómeno El Niño de 1925 no superaban entonces esa cifra .

Entre 1940 y 1972 se pasó de una población predominantemente rural (65\%) a una mayoritariamente urbana ( $60 \%$ ). En 1940 solo 5 departamentos tenían población predominantemente urbana mientras que en 1972 eran 13.

Entre 1900 y 1969 destacan los desastres desencadenados por las epidemias entre 1903 y 1930, las inundaciones asociadas al Fenómeno El Niño en 1925, los aluviones de 1941 y 1962 en la Cordillera Blanca, los terremotos en el Callejón de Conchucos (1946) y Cusco (1950) y las sequías en el sur del país a fines de la década de 1950 e inicios de 1960.

A partir de la década de 1940, el Estado logra mayor eficiencia en la repuesta a emergencias y la reconstrucción en áreas urbanas, lo que se ve facilitado por la mejora de las vías de comunicación y la mayor disponibilidad económica. En contraste, las poblaciones rurales y de los poblados de la sierra y selva no reciben la atención estatal (Rivera, 1983; Municipalidad de Satipo, 2020). Un aspecto destacable es el relativo a las medidas de reducción de riesgo, aunque asumidas después de un desastre, fue el monitoreo de las lagunas y glaciales en Ancash después del aluvión que afectó Huaraz en 1941 o las iniciativas de proyectos de irrigación como el de Chira-Piura a raíz de la sequía que afectó dicho departamento.

La preocupación de las autoridades por "El Niño" y otros fenómenos climáticos extremos se centrará en la década de 1960 en sus efectos sobre la pesca de anchoveta. Sin embargo serán ignoradas e incluso reprimidas las advertencias de algunos científicos sobre nuevos peligros de un aluvión, como el que sucederá años después en Yungay. 
La creación de la Dirección de Salubridad Pública en 1903, la primera agencia nacional de salud, evidencia un cambio significativo estatal para enfrentar la peste bubónica que afectó a las principales ciudades hasta 1930; también la formación de lazaretos (lugares donde se confinaba a la población enferma) en las principales ciudades, de estaciones sanitarias en los puertos, la generalización de las desinfecciones y diversas disposiciones sanitarias. A pesar de ello, la población se resistía a la intervención médica mediante la negación de la existencia de la enfermedad, la huida de los lugares afectados y de los lazaretos y pequeñas revueltas que influyeron en el autoritarismo oficial.

La gripe española se desató a principios de 1918 y se prolongó hasta 1920 en tres olas que afectó a 500 millones de personas que en esa época constituían el $30 \%$ de la población mundial y causó entre 50 y 100 millones de muertes, en su mayoría gente entre 20 y 40 años de edad que sentían debilidad y tenían neumonía, problemas estomacales, dificultades para respirar, confusión y fiebre y morían en cuestión de horas. En todo el mundo se trató de limitar los contagios mediante medidas muy similares a las de la actual pandemia. ${ }^{4}$

El impacto de la gripe española en el Perú se conoció recién en julio del 2011 cuando se difundió un estudio (Revista Vaccine, 2011), donde se precisó que una primera ola de gripe se inició en Lima en julio y se prolongó hasta septiembre de 1918, generando alta mortalidad. La segunda ola se presentó entre noviembre de 1918 y febrero de 1919 y se extendió casi al mismo tiempo a lquitos y Trujillo. La tercera ola ocurrió entre enero y marzo de 1920 en Lima y de julio a octubre de 1920 en Ica. No se cuenta con datos de personas fallecidas, pero en otros países de Sudamérica superaron las 30 mil.

La epidemia fue una oportunidad para convencer a los poderes públicos y a parte de la población de la importancia que tenían los profesionales de la salud y los métodos de higiene. La Conferencia de Paz de París, que tuvo lugar en 1919, sentó las bases del sistema moderno que existe para el control global de crisis sanitarias.

El Fenómeno El Niño de 1925 generó lluvias que provocaron inundaciones en ciudades como Piura y Tumbes con menos de 20 mil habitantes y otras como Lima, Trujillo, Chiclayo e Ica que tenían una población que superaba esta cifra; José Carlos Mariátegui advirtió sobre su impacto en la salud y en la economía, y denunció que los hacendados impedían la atención de las familias afectadas por enfermedades y epidemias que vivían en las haciendas (Mariátegui, tomo I, 1994: 315).

$4 \quad$ En un estudio comparativo de los protocolos seguidos en 1918 en 43 ciudades se concluye que todas las ciudades aplicaron al menos una de estas tres medidas: a) suspensión de las clases escolares, b) prohibición de aglomeraciones y c) aislamientos o cuarentenas. 
En contraste con la débil respuesta del gobierno nacional ante las inundaciones de 1925, se produjo en el país una importante respuesta local. La defensa de las principales ciudades y localidades se hizo de forma voluntaria. En el campo bajo la autoridad de los hacendados, los asalariados agrícolas tuvieron una participación activa en la protección de fundos y haciendas.

En diciembre de 1941, en el contexto de un fenómeno El Niño intenso una gigantesca lengua de nieve se desgajó del nevado Palcaraju, y provocó el desborde sucesivo de dos lagos generando un flujo de agua de quince metros de altura que bajó arrasando ocho poblados y parte de Huaraz.

El alcalde, supo impartir las órdenes, para evitar el acaparamiento y los saqueos y para atender a los heridos. “Con la policía trabajó tesoneramente la Guardia Urbana que voluntariamente se alistó para cooperar. Formaron parte de ella casi todos nuestros artesanos, miembros de las sociedades de tiro y la juventud" (MINAM, s/f: 46).

El Congreso aprobó un crédito extraordinario para reconstruir el tramo del ferrocarril destruido y para la canalización del río Quillcay. Además, hubo un fondo que fue redistribuido seis meses después por la Junta Pro Damnificados en Huaraz de acuerdo a un empadronamiento previo.

A raíz de este desastre se constituye la ahora denominada Unidad de Glaciología y Recursos Hídricos, que tiene como función principal inventariar y vigilar las lagunas de la Cordillera Blanca y por decreto del 18 de noviembre de 1942 se dispuso la creación y organización de la Defensa Civil Nacional.

El 10 de noviembre de 1946 a las 12:53, ocurrió un sismo superficial de magnitud 7,3 con epicentro a 7 kilómetros al sur del distrito de Conchucos (provincia de Sihuas, Ancash). La destrucción era tal, y sobre todo en la provincia de Sihuas, que causaron la muerte de por lo menos 2,500 personas (IGP, 2013, boletín152). Este terremoto puso al descubierto una falla tectónica, hoy conocida como falla de Quiches.

Después que cesaron las réplicas, la población de Sihuas empezó a reconstruir sus casas de adobe (Bisso, 1946, hito 3). Sólo los sobrevivientes del destruido pueblo de Chingalpo decidieron reubicarse como ya lo habían hecho antes cuando el pueblo fue sepultado por el derrumbe de la Meseta de Mollepata ${ }^{5}$.

El domingo 21 de mayo de 1950 a las 13:39, un sismo que tuvo sólo una duración de 6 o 7 segundos causó gran destrucción en Cusco y obligó a sus habitantes a correr buscando el espacio abierto, lejos de las paredes y los techos que se des-

5 La historia desconocida de Chingalpo 1704-1958. Municipalidad distrital de Chingalpo, Facebook 28 de marzo de 2019. 
plomaron (Tamayo Herrera,1978: 161-168). 3000 casas quedaron destruidas, solo 1200 quedaron en condiciones de ser habitadas.

Los damnificados pernoctaron nueve noches en la plaza y los parques hasta que se instalaron 1642 carpas de distintos tamaños. Veinte mil personas abandonaron Cusco en automóviles, camiones y ómnibus, por tren o pie, dirigiéndose hacia los pueblos cercanos y después a Lima y Arequipa.

Se creó la Junta de Reconstrucción y Fomento Industrial del Cuzco que fue precursora de las posteriores corporaciones departamentales, aunque en este caso tuvo la dirección en Lima y lo básico de sus estructura en el Cuzco. Entre junio de 1951 y septiembre de 1973, se reconstruyeron con préstamos estatales 341 edificios pero la falta de una reglamentación adecuada impidió un control eficaz del modelo arquitectónico a seguir y del monto máximo del préstamo, resultando que 30 propietarios privados recibieron sumas por encima de 1'000,000 de soles de la época con las que pudieron construir edificios de grandes dimensiones ( Tamayo, 2010: 170 y siguientes).

El 10 de enero de 1962 a las 18:05 se desprendió una enorme cornisa de hielo del pico norte del Huascarán y generó un alud que sepultó Ranrahirca y varios pueblos. Desaparecieron 4000 habitantes.

A raíz de este desastre, cientos de inmigrantes llegaron a Yungay porque lo consideraban "La ciudad más segura del Callejón de Huaylas". La provincia financió la ampliación de la infraestructura y servicios para los nuevos vecinos. Sin embargo los científicos identificaron una inestabilidad masiva en el campo de hielo sobre Yungay, lo que se informó al gobierno y apareció en la prensa nacional, pero el gobierno emitió una orden a los científicos para retractarse o enfrentar la prisión (Carey, 2005: 130).

\section{Los desastres y la Defensa Civil: 1970-1986}

El desastre del 31 de mayo de 1970 constituye un hito muy relevante por la letalidad y destrucción generada. Las experiencias para responder a una emergencia en un territorio donde se carecía de información y los cambios que se hicieron durante la reconstrucción posibilitaron reducir la vulnerabilidad de algunas ciudades y centros poblados mediante una mejor distribución del espacio urbano y un cambio en los patrones de construcción. En contraste los cambios en las viviendas de las poblaciones rurales alto-andinas fueron mucho menores.

El desastre se inició con un sismo de magnitud $7.8 \mathrm{M}_{\mathrm{w}}$ en la escala de magnitud de momento, sentido en toda la costa y sierra del departamento de Áncash y 
del norte peruano, seguido de un aluvión que sepultó la ciudad de Yungay. En las ciudades y los pueblos medianos de la sierra y la costa de Ancash, los edificios de adobe y hasta los de material noble comenzaron a destruirse aproximadamente 15 segundos después de iniciado el terremoto. Las tejas de los techos comenzaron a caer, convirtiéndose en proyectiles peligrosos. Las paredes de las casas se tambaleaban y caían rápidamente encima de los habitantes.

La ciudad de Huaraz se destruyó en un $97 \%$ y fallecieron 10 mil habitantes. Las ciudades de Yungay y Ranrahirca con sus cerca de 25 mil habitantes fueron sepultadas por un aluvión. Los aludes y derrumbes obstaculizaron caminos y carreteras y represaron partes del río Santa. El ferrocarril que unía a Chimbote con Huallanca desapareció y solo una pequeña pista de aterrizaje en Anta pudo ser rehabilitada (Oliver-Smith, 1986: 156).

Debido a que los pueblos de la zona estaban muy alejados entre sí y con pocos medios de comunicación fue muy importante la labor de los radioaficionados. Muchos de los víveres fueron repartidos en paracaídas. Un buque cisterna trasladó 1000 metros cúbicos de agua a Chimbote dos días después del sismo. La solidaridad internacional fue inmensa.

El Ministerio de Vivienda estableció un comité de emergencia para evaluar los daños, facilitar hospedaje temporal y restablecer los servicios esenciales de agua, saneamiento y otros (UNDRO, 1982: 71). El problema de la vivienda se resolvió temporalmente con carpas y albergues, mientras se iniciaba la operación Techo, con la que se construiría 1439 albergues multifamiliares. Los alojamientos de emergencia estaban formados por una estructura de metal y chapas de hierro onduladas que posteriormente se utilizaron en la reconstrucción de viviendas.

Se creó el Comité de Reconstrucción y Rehabilitación de la Zona Afectada (CRYRZA), el que dos años después, fue reemplazado por el Organismo para el Desarrollo de la Zona Afectada de Ancash (ORDEZA).

En agosto, los funcionarios oficiales impulsaron la reubicación y reconstrucción de más largo plazo. Aproximadamente, de los 27000 sobrevivientes del terremoto y avalancha en toda la provincia de Yungay, se estimó que entre 4000 a 5000 habían escapado a Lima (Carey, 2014: 209).

En Huaraz se hicieron estudios de microzonificación sísmica, se expropiaron los terrenos y propiedades más extensas para distribuirlos entre los que no poseían propiedades, y se ampliaron las calles del centro, pero en las zonas periféricas y en el resto del Callejón de Huaylas se siguieron construyendo las casas igual que antes del terremoto. 
Se recomendó revisar las obras de las lagunas incluida Palcacocha y se procedió a la construcción de un dique de 8 metros de altura que se terminó en 1974. Sin embargo, en el 2006 la laguna había crecido 34 veces su volumen (supera los 17 millones de metros cúbicos), lo que aumentaba fuertemente los efectos que podrían tener posibles avalanchas de hielo, deslizamiento de tierras o desplome de masas rocosas sobre la laguna, que de desbordarse estarían amenazando la vida de más de 50 mil habitantes de la ciudad de Huaraz. (MINAM, 2014: 68-70).

A raíz de este desastre y la creación posterior del Sistema Nacional de Defensa Civil, se produce un cambio sustantivo en el manejo de los desastres porque el Estado centra más su atención hacia la seguridad de la gente ( Franco, Eduardo y Zilver 1996:359-372; Ferradas, 2000:68-69) y en la descentralización de la respuesta a desastres. Eran, también, tiempos donde el Gobierno promovía la organización y participación social orientándola hacia el apoyo a las reformas que se implementaron hasta $1974 .{ }^{6}$

A partir del año 1972, suceden algunos desastres que devienen en demandas y protestas masivas, y otros, como los desencadenados por un sismo en Lima y El Niño 1982-1983 que permiten extraer lecciones significativas.

En marzo de 1972, durante la presencia de el "Fenómeno El Niño olvidado" (SENAMHI, 2014: 1) en el que no se tomaron medidas para limitar la captura de la anchoveta facilitando con ello su virtual desaparición, se desborda el río Lacramarca e inunda el casco urbano de Chimbote, incluida la plaza de armas y las avenidas principales, cientos de viviendas dañadas, fueron posteriormente demolidas. Esto ocurre en un contexto de desempleo generado por la interrupción de la actividad pesquera y deriva en una protesta popular y enfrentamientos que motivan la declaratoria de emergencia en Chimbote. Más que por la falta de la ayuda humanitaria, la gente reclamaba por la pérdida de sus medios de vida.

Los efectos negativos en la producción pesquera se prolongaron por varios años y también influyeron en la estabilidad económica de los siguientes períodos, en razón del agotamiento de divisas, a las que contribuía fuertemente la pesca.

A raíz de la inundación de Chimbote se procedió, años después, a desviar el cauce del río para que no pasara por el centro de la ciudad; de esta manera algunos asentamientos humanos se transformaron en vulnerables a las inundaciones de un río que anteriormente se encontraba bastante alejado. Es por ello que en 1998, se inunda justamente el asentamiento Villa María en Nuevo Chimbote.

6 Franco y Zilver analizan los antecedentes de la formación del Sistema Nacional de Defensa Civil; sus congruencias e incongruencias. 
El jueves 3 de octubre de 1974 a las 09:31 un sismo de magnitud 7,5 devino en un desastre en Lima y hacia el sur hasta Pisco y se sintió en Trujillo y Arequipa.

En Lima, los mayores daños ocurrieron en La Molina (VIII-IX MMI de intensidad), donde dos edificios de concreto armado colapsaron y otros resultaron muy dañados (Barreau, Lourane y Peña s/f: 27 y 28). También sufrieron graves daños algunas zonas del Cercado de Lima y del Rímac, así como los distritos de Barranco y Chorrillos (VII-VIII), además de la zona de acantilados y los distritos de San Miguel, Magdalena y Miraflores (VIII). El Callao también fue severamente afectado, sintiéndose el sismo con mayor intensidad, en los distritos de La Punta y La Perla (VII-VIII). Las construcciones de concreto sufrieron graves daños y las de adobe colapsaron.

El gobierno expidió un decreto que prohibió el desalojo de los ocupantes de viviendas dañadas y asumió la tarea de apoyar a las familias afectadas reubicándolas y apoyando la construcción en nuevos barrios, como sucedió con los vecinos del jirón Amazonas, en el centro de Lima, que fueron reubicados en San Juan de Lurigancho (El Comercio, 3 octubre 2019).

En 1982 y 1983 ocurre el Fenómeno El Niño, considerado el más intenso en 450 años, en un contexto de crisis económica que la agudizó aún más. La región sur alto-andina fue afectada por una sequía que causó grandes pérdidas en los cultivos y en la crianza de animales. Los pobladores del altiplano estuvieron al borde de una hambruna generalizada y se produjo una gran emigración hacia otras zonas e incluso hacia otros países para poder subsistir (Rocha, 2007: 33).

En la costa norte y central se produjeron grandes inundaciones que causaron la destrucción de viviendas, cultivos e infraestructura social en general y se presentó un notable incremento de las enfermedades respiratorias, gastrointestinales y dermatológicas, además de otras como la malaria, que ocasionaron un fuerte aumento de la mortalidad, especialmente infantil (Ferradas, 2000: 72 y 73).

El Instituto Nacional de Planificación estimó en dos millones setecientos mil las personas afectadas en el Perú aunque otras fuentes las estimaron en seis millones, lo que representaba la tercera parte de la población del país. El impacto económico fue estimado en mil millones de dólares y parte de sus efectos incidieron en 1983 en la disminución del PBI en 12,3\%, la inflación del 125\% y en la ausencia del crecimiento económico en el siguiente quinquenio (CONCYTEC,1,984: 647).

Los efectos de los desastres y la crisis fueron mitigados por la organización comunitaria de la Sociedad Civil; principalmente en la costa que tuvieron un papel determinante para la atención de las necesidades de la gente y para la rehabilitación de los asentamientos. En Piura se organizó el Frente de Defensa del Pueblo, 
que lo integraban un centenar de organizaciones( SEPIA VIII, 2000: 137-141 y196; Ferradas, 2000: 83).

El Programa de Emergencia del Sector Agrario incluyó la reconstrucción de la infraestructura de riego; el refinanciamiento del $60 \%$ de los préstamos a diciembre de 1983 del Banco Agrario a plazos de hasta 10 años; nuevos créditos para reconstruir la infraestructura a nivel de predio y para capital de trabajo y un sistema promocional de precios de algunos productos agrícolas (Rocha: 34-36).

Se creó el Instituto Nacional de Desarrollo (INADE) para coordinar y conducir los Proyectos Hidráulicos y Especiales en Sierra y Selva y el Programa de Rehabilitación y Reconstrucción. La ejecución estuvo a cargo de las Corporaciones Departamentales de Desarrollo (CORDES), a través de empresas consultoras y constructoras, la red vial nacional que fue encargada al Ministerio de Transportes y Comunicaciones.

La vivienda tuvo poca atención, salvo en la prioridad otorgada a los damnificados en los programas estatales de crédito para vivienda y en la ejecución de algunos proyectos habitacionales utilizando el adobe reforzado con caña recomendado por ININVI (Montoro y Ferradas, 2005: 28).

Durante el verano 1985-1986, la zona de Puno sufrió fuertes inundaciones debido a un incremento del nivel del lago Titicaca, afectando los cultivos de 146 comunidades y destruyendo 2173 viviendas. También cayeron los ingresos de la fibra de alpaca cuya producción estaba, fundamentalmente, en manos de productores campesinos. Tras los desbordes y la caída de los precios vino la profusión de eventos y asambleas con debates sobre la reestructuración de la propiedad de las tierras y el futuro regional liderados por la Federación de Campesinos de Puno (Comisión de la Verdad, 2003: 525). En julio de 1986, se produjo un paro departamental y un gigantesco mitin organizado por la Federación Única de Campesinos de Melgar (FUCAM) demandando la distribución de tierras. Con el renovado apoyo de las iglesias y organizaciones gremiales y profesionales de la capital departamental, el movimiento alcanzó su éxito con el anuncio en el mensaje a la nación de Fiestas Patrias, el 28 de julio de 1986, que 1100000 hectáreas serían distribuidas entre las comunidades de Puno.

\section{Los nuevos enfoques para reducir los riesgos y los desastres 1987-2007}

En el año 1990 en un evento organizado por Naciones Unidas en Japón se acuerda el Marco de Acción de Hyogo, en el que se incorporan aspectos claves para la"reducción de desastres"y enfoques centrados en los derechos de las personas. También 
fueron difundidos los estándares mínimos de ayuda humanitaria que fueran elaborados por numerosas organizaciones especializadas en la respuesta a desastres.

Entre los desastres ocurridos entre 1987 y el año 2007 destacamos: el desencadenado por los huaycos en Chosica en 1987; la epidemia del cólera a inicios de la década de 1,990; los terremotos en 1990 y 1991 en el Alto Mayo, en 1996 en Nazca, en el 2007 en el Sur Chico y las inundaciones derivadas del FEN 1997-1998.

Estos desastres permitieron constatar la insuficiente delimitación de responsabilidades que generaba la duplicidad y los conflictos de competencia de las instituciones gubernamentales en plenas emergencias; el debilitamiento de las organizaciones de la población; un aumento de la solidaridad pública inducida por los medios de comunicación; la ausencia de una perspectiva integral de las cuencas para reducir los riesgos de inundaciones y la falta de difusión y aplicación de estudios de riesgos existentes.

La reconstrucción constituyó una experiencia aparte en los casos de Alto Mayo y Nazca. El Estado centrado más en la reconstrucción de infraestructura y servicios públicos se implicó limitadamente en la reconstrucción de viviendas en ámbitos urbanos, mientras que las ONG implementaban programas en los asentamientos populares y en los poblados rurales (Vischer, 2008).

En 1987 durante el Fenómeno El Niño (FEN) moderado se producen huaicos en Chosica donde un centenar de persona fueron consideradas como desaparecidas, se destruyeron centenares de viviendas y se bloqueó durante dos semanas la carretera Central afectando el comercio entre Lima y el centro del país y el abastecimiento de alimentos perecibles.

Los principales problemas enfrentados en la emergencia fueron el abastecimiento de agua, la ayuda a los damnificados, la limpieza de las vías principales y la seguridad ciudadana ante lo cual se declaró el toque de queda. El agua fue reabastecida 40 horas después, existiendo mucha desorganización en la distribución de alimentos y ropa a los damnificados que se alojaron en carpas, casas de vecinos y locales públicos y religiosos (IDMA, 1987: 180).

En los meses siguientes se dio una gran movilización de la población y las instituciones de los asentamientos más afectados de Chosica. Se impulsaron acciones que posibilitaron la delimitación y apertura de los cauces de las quebradas que habían sido invadidos por las viviendas, la implementación de medidas para reducir los riesgos como el no dotar de servicio de electricidad a las viviendas que se construyeran en las zonas inseguras, el habilitar caminos de acceso y lugares de refugio ante posibles huaycos, la mejora y protección de los sistemas de agua potable, el control o estabilización de laderas con la participación de los progra- 
mas sociales (Vaso de leche, empleo temporal) y la organización de jóvenes en las escuelas y la comunidad). Todo ello mereció el reconocimiento de Naciones Unidas que en la Conferencia de Yokohama de 1995 que puso como ejemplo de prevención comunitaria de desastres a la población de Chosica.

En 1990 y 1991 se produjeron dos terremotos de 6 y 6,2 grados de magnitud que generaron desastres en el Alto Mayo. A las 21:34 del martes 29 de mayo de 1990 un sismo afectó a las provincias de Rioja y Moyobamba en la Región San Martín. Se estima 200 fallecidos, 1200 heridos y 250,000 damnificados. El 4 de abril de 1991, otro sismo volvió a afectar a la misma región golpeando a las pocas localidades que no habían sufrido el impacto del anterior. El Instituto Geofísico del Perú, reportó 100 fallecidos.

EI INDECI llegó con ayuda para la emergencia y para la evaluación de los daños y las necesidades. Fue con su llegada que se constituyeron los comités provinciales y distritales de Defensa Civil. La reconstrucción gubernamental se centró fundamentalmente en la infraestructura social afectada y las ONG implementaron programas participativos de construcción de viviendas de quincha que reemplazaron las anteriores que eran predominantemente de adobe, dada la inexistencia de producción de ladrillo y cemento en ese entonces en San Martín.

En el año 1991 se produjo la epidemia del cólera que afectó a centenares de miles y causó la muerte de 2909 personas y puso en evidencia la precariedad de los sistemas de agua y desagüe y de salud, agravados por la política de shock implementada el año anterior que afectaba a los más pobres.

En una entrevista realizada antes de la epidemia, al entonces ministro de Salud, Vidal Layseca, señaló que, al recibir el cargo, los hospitales de Lima y provincias"no tenían ni para combustibles, ni para alimentos, no tenían para medicamentos, es decir prácticamente no tenían para nada" (Chasqui, 1991: 38-40).

El Estado difundió la idea de que existía una responsabilidad individual al contraer la enfermedad. La población había comprendido que la salud era una responsabilidad de los individuos antes que del Estado y, por ello, existía la convicción de que no se podía exigir mucho en esta materia; los discursos liberales habían promovido la idea de que el Gobierno tenía pocas obligaciones y no debía intervenir. Frente a esta débil actuación estatal, destaca el rol que jugaron las instituciones de la Sociedad Civil, especialmente las rondas campesinas en Cajamarca, los comedores populares y los comités de vaso de leche en las ciudades. (Cueto, 1997: 215).

El 12 de noviembre de 1996, a las 17:00 horas se produjo un sismo de 7,7 grados, con epicentro localizado a $135 \mathrm{~km}$ al sur-oeste de la ciudad de Nazca. Aunque tuvo una intensidad moderada, el $75 \%$ de las casas de adobe en las provincias de 
Nazca y Caravelí sufrieron daños severos o colapsaron. También se observó daños en edificaciones de concreto armado de las escuelas construidas recientemente, debido a deficiencias en el diseño y construcción, aunque existieron también denuncias contra las empresas constructoras. En un estudio posterior (San Bartolomé, Rivera, Durán, Muñoz y Quiun, 2015: 84.) se propuso una técnica de reforzamiento para edificaciones existentes con problemas de columna corta, que fue el caso de las escuelas construidas en la década de 1990.

El movimiento sísmico ocasionó, además, el derrumbe de los depósitos de relaves en el campamento minero de Otapara localizado en el distrito de Acarí y causó la destrucción de cultivos y zonas agrícolas ubicados en las áreas ribereñas del río Acarí y la contaminación de sus aguas.

Inmediatamente después de ocurrido el sismo, la población y las autoridades locales organizaron la respuesta con el apoyo de una emisora local. Se llamó a la población a formar comités en cada calle a fin de impedir posibles robos, evitar derrumbes y accidentes, recuperar algunos bienes sepultados y acceder de manera organizada a la ayuda (incluida el agua) que estaba siendo canalizada por la municipalidad, de acuerdo con la normatividad de Defensa Civil. Sin embargo, con la llegada, desde Lima, de un contingente de funcionarios y camiones del ejército se inició un conflicto de competencias, que solo fue resuelto por la decisión formal del Gobierno Central de asumir tal competencia. Los conflictos de competencia entre las distintas instancias del Estado ha sido una constante y en lo fundamental evidencia la imposición de las instituciones centrales en relación con los gobiernos regionales y locales y el afán de protagonismo existente.

En la ciudad de Nazca, el desastre reveló la generalizada informalidad en la tenencia de los suelos con ocupantes que acreditaban posesión, pero que no exhibían títulos de propiedad. En el centro de Nazca la mayoría de viviendas eran compartidas con inquilinos, lo que generó demandas diferentes ya que los posesionarios exigían al Gobierno apoyo para reconstruir sus viviendas, pero no reconocían a los inquilinos el derecho a quedarse en ellas. Dicha situación planteó tres problemas por resolver: el saneamiento físico-legal de los suelos, la relocalización de los inquilinos y la reconstrucción de viviendas. (Montoro, 2005).

Numerosas viviendas fueron edificadas con apoyo gubernamental y de las ONG (Vischer, 2008: 9). Sin embargo, pocos años después, en plena ocurrencia del fenómeno de El Niño, cerca de 50 viviendas que habían sido construidas en Cajuca previo saneamiento físico y legal apoyado por la Empresa Nacional de Edificaciones (ENACE) y la Comisión de Formalización de la Propiedad Informal (COFOPRI) fueron 
destruidas, esta vez por efecto del flujo de lodo y piedras que se precipitó por la quebrada donde se ubicaba el asentamiento.

En las áreas rurales las familias afectadas no tenían establecido legalmente un derecho privado con respecto a los terrenos que ocupaban; sin embargo, las juntas de productores de cada poblado reconocían a los parceleros el derecho de ocupación de un lote de terreno dentro del pueblo para reconstruir su vivienda, pero no admitía en muchos casos tal derecho a los campesinos sin tierras o jornaleros que también habían perdido sus viviendas. Esto planteó también resolver tres problemas en las áreas rurales: el saneamiento físico-legal de los suelos de los asentamientos rurales, el acceso al suelo para vivienda de los campesinos jornaleros y la reconstrucción de las viviendas rurales (Predes 2005 y Vischer, 2008: 9).

Las ONG intervinieron con programas de reconstrucción focalizados principalmente en las zonas rurales y pequeños centros poblados, promovieron la participación de los damnificados, el fortalecimiento de las organizaciones y el uso de la quincha y el adobe mejorados.

En el proceso de reconstrucción en Nazca, la gestión territorial que recaía en antiguos dirigentes de las cooperativas, fue sustituida por organizaciones lideradas en su mayoría por mujeres y con más participación de los vecinos.

El fenómeno El Niño1997-1998 constituye un escenario diferente a los anteriores en la medida en que se contaba con información previa y por tanto con tiempo para prepararse antes de la emergencia. El esfuerzo del Estado se centró principalmente en las denominadas obras de prevención que en realidad eran en su mayor parte medidas de emergencia ante la inminente presencia de El Niño.

Las pérdidas económicas fueron extremadamente significativas porque se creyó erradamente que todos los Fenómenos El Niño eran iguales y, por tanto, se ignoró los posibles efectos en Ica que fue la ciudad más castigada y se tomaron medidas contra la sequía en la Sierra Sur que fue más bien afectada por huaycos e inundaciones. Tampoco se propició la organización de la población ante los desastres; el énfasis fue puesto en difundir desde los medios de comunicación algunos mensajes y el notificar a las poblaciones ubicadas en zonas de alto riesgo para que se reubiquen.

La inundación en Ica afectó a miles de personas porque se destruyeron centenares de viviendas y se interrumpieron durante semanas las actividades y servicios; algunos testimonios de pobladores dan cuenta de que en los primeros días la gente se turnaba para dormir sobre las aceras no inundadas. Se produjo entonces una migración de numerosas personas hacia Lima y otras ciudades donde recibieron el apoyo de familiares (Ferradas, 2000: 96). 
El Gobierno central tuvo como primera prioridad rehabilitar la red vial; la segunda prioridad fue la vivienda. El último lugar lo ocupaba el sector agricultura, con el $4 \%$ del total de recursos invertidos durante la fase de rehabilitación. (SEPIA VIII, 2000: 214-216) (PROMPERÚ, 1999: 337-338).

En las ciudades se constituyeron diversos mecanismo de articulación de las organizaciones existentes. En Ica se formó una Coordinadora Vecinal de Familias Damnificadas, y posteriormente el Frente Cívico por la Reconstrucción y el Desarrollo con representantes de damnificados y de organizaciones vecinales y gremiales.

En Lambayeque se organizó la Acción Ciudadana y en Piura la Coordinadora Interinstitucional para el Desarrollo y el Grupo de Gestión del Riesgo, integradas por instituciones públicas y privadas; y se organizaron grupos de jóvenes voluntarios que brindaron apoyo a las comunidades afectadas.

En los diversos eventos realizados con las organizaciones sociales, se destacó la importancia de los planes de mediano y largo plazo de reordenamiento urbano y rural; de una visión integral de cuencas; de las campañas de información, organización y educación comunitarias; y de las redes sociales de prevención de desastres. Entre las propuestas destacaron: el empleo temporal orientado a generar ingresos para los damnificados mediante las actividades de rehabilitación y reconstrucción; implicar a los colegios profesionales y universidades en la reconstrucción; incorporar estrategias de prevención y mitigación de desastres en los programas y proyectos de desarrollo; la asistencia técnica estatal para la rehabilitación de infraestructura productiva priorizando al campesinado pobre e incorporando tecnologías apropiadas; y el crédito estatal y privado orientado a generar capitales de trabajo; la creación de un Fondo para la investigación científica y tecnológica en relación con los eventos peligrosos y para la educación en prevención de desastres; y el acceso al crédito promocional en caso de pérdida de vivienda por desastres.

Tales propuestas no fueron en su mayor parte acogidas por el Gobierno que optó por sus propias iniciativas de reconstrucción y el Programa Ciudades Sostenibles que entre el año 1998 y el 2009 elaboró estudios de riesgo en 131 ciudades pero cuya aplicación en los planes municipales ha sido insuficiente.

El 23 de junio 2001 a las 15:33 ocurrió un sismo con epicentro localizado en el mar frente a Ocoña y con magnitud de 8,4 Mw, que afectó los departamentos de Arequipa, Moquegua, Tacna, Ayacucho y Apurímac. El gobierno estableció una junta de apoyo multisectorial para atender a los damnificados del sismo y en la reconstrucción destacó el liderazgo de la Municipalidad de Moquegua que junto con otros gobiernos locales vieron la posibilidad de reducir los riesgos en el proceso de reconstrucción asignando terrenos más apropiados para ello y gestionando 
recursos de la cooperación internacional y del Gobierno para dotar de servicios e implementar algunos programas de vivienda

En la primera década del siglo XXI los medios de comunicación y el Gobierno prestaron atención a las emergencias y desastres recurrentes en las zonas sur alto andinas debido a las heladas. El gobierno por gestión del INDECI ha declarado varios años en emergencia a varias provincias del sur andino, cuyo número varía por el grado de intensidad y extensión territorial de los descensos de temperatura que se presenta recurrentemente en los meses de junio y julio (INDECI, 2009: 23).

El miércoles 15 de agosto de 2007 a las 17:40 horas ocurrió un sismo de magnitud 7,9 que dejó un saldo de 519 muertos, 1291 heridos, cerca de 90 mil viviendas destruidas o con grave daño, y la afectación e interrupción de la infraestructura y los servicios públicos y privados principalmente en Cañete, Chincha y Pisco (INDECl, 2009: 23).

Este desastre evidenció problemas en la respuesta y en la reconstrucción. Entre estos se encuentran: las fallas en la comunicación que llevó a errores de apreciación del Presidente de la República; inexistencia de las instancias municipales de defensa civil $^{7}$ e incapacidad inicial para responder a la emergencia (INDECI, 2009: 24); carencia de información inicial confiable sobre las personas damnificadas y afectadas; inexistencia de títulos de propiedad saneados que complicó la atención de la ayuda y la identificación de beneficiarios en la reconstrucción; corrupción y falta de asesoría técnica en los programas de reconstrucción gubernamental.

Los problemas fueron afrontados parcialmente mediante la intervención del INDECI , instituciones nacionales, las ONG y las organizaciones de la población (particularmente de mujeres) lo que posibilitó mejorar la atención de las necesidades inmediatas; se instalaron un centenar de albergues y miles de carpas (INDECI, 2009:48). Mientras que los pobladores afectados en las ciudades esperaban ayuda en alimentos, abrigo y techo; los de las zonas rurales solicitaban principalmente herramientas manuales para reconstruir sus canales de regadío colapsados. (INDECl, 2009: 25).

Los pobladores de las zonas rurales reconstruyeron sus canales de riego y viviendas, la población en algunos centros poblados y ciudades lo hicieron con el apoyo de las ONG y el Estado. Mientras que en el primer caso se dio asistencia técnica y se promovió la participación de los pobladores en la reconstrucción de viviendas y tecnologías como la quincha y el adobe mejorado; en el segundo caso el Estado dio bonos para que la población comprara materiales de construcción,

7 Se constató que el personal previamente capacitado en la zona por el INDECl ya no estaba en funciones y que otras autoridades, elegidas en noviembre de 2006, los había reemplazado. 
lo que devino en denuncias de corrupción y en los casos en que se reconstruyeron viviendas, éstas no tuvieron la asesoría técnica necesaria ni el control de las autoridades locales. El Estado asumió también la reconstrucción de la infraestructural social dañada, lo que en algunos casos devino en nuevas denuncias de corrupción y en la postergación de parte de las obras por más de una década.

\section{EI SINAGERD y los retos de la Gestión de Riesgo (GRD): 2011-2020}

En el año 2011 se promulga la ley de creación del Sistema Nacional de Gestión de Riesgo de Desastres (SINAGERD) y en los meses siguientes un conjunto de normas complementarias e instrumentos entre los que destacan el Reglamento de la Ley, la Política y el Plan Nacional de Gestión de Riesgo 2014 -2021. Entre los principales cambios destacan el que los Gobiernos Locales y Regionales, así como los Sectores, son los responsables de la ejecución de las políticas de GRD que incluyen, además de la preparación y respuesta a desastres (especialidad del INDECI), la reducción de los riesgos y el evitar que se generen nuevos riesgos en los procesos de desarrollo (para lo que se crea el Centro Nacional de Estimación y Prevención de Riesgos de Desastres -CENEPRED) como entidad especializada). El ente rector de la GRD es la Presidencia del Consejo de Ministros y tienen roles claves el CEPLAN y el Ministerio de Economía y Finanzas para la incorporación de la GRD en la planificación del desarrollo y para el diseño de una estrategia financiera que asigne los recursos a las diferentes entidades ejecutoras y especializadas.

La nueva legislación tiene como referente cercano los problemas identificados durante el desastres del año 2007, la debilidad de los mecanismos para reducir los riesgos y las iniciativas internacionales y regionales tendientes a promover la gestión de riesgo de desastres.

En la actualidad, el SINAGERD tiene la mayor implicación de los sectores e instancias regionales y locales, favorecida por los programas presupuestales; la mejora y complementariedad de los sistemas de información, en particular los centros de operaciones de emergencias; $y$ el mayor protagonismo de las Fuerzas Armadas en la atención de las emergencias.

EI SINAGERD tiende a centrarse en los fenómenos y la vulnerabilidad de las construcciones sin considerar suficientemente los procesos sociales que generan los riesgos y las estrategias sociales de GRD. Tampoco ha logrado la implicación de las universidades; tiene limitados mecanismos y espacios de participación social ; carece de sanciones efectivas a las personas y empresas que generan o incremen- 
tan los riesgos; y persiste la lentitud de los procedimientos para la reconstrucción derivados de la normativa.

Entre los desastres ocurridos desde la creación de la Ley del SINAGERD destacamos los desencadenados por las inundaciones del 2017 y las heladas y friajes de los años 2018 y 2019 y la actual pandemia. Todo ello en un contexto de disminución del crecimiento económico y de crisis política.

En el primer trimestre del año 2017 se produjeron lluvias e inundaciones a lo largo de la Costa desencadenando desastres que causaron 101 muertos, dañaron cientos de miles de casas ( 55000 fueron destruidas) y cientos de escuelas y centros de salud; $y$ destruyeron cientos de kms de canales de riego y carreteras. Las pérdidas económicas fueron estimadas en 3125 millones de dólares .

A raíz de estos desastres se generó una gran movilización nacional e internacional que permitió masivas donaciones para los damnificados, las que fueron trasladadas y distribuidas por las Fuerzas Armadas y por las autoridades. También, se dio la respuesta regional y sectorial destinando rápidamente fondos para la compra de motobombas, limpieza de cauces, e instalación de módulos temporales; y una mejor articulación de la ayuda de las ONG Soluciones Prácticas, PREDES, Ayuda en Acción, Plan Internacional y World Vision con el liderazgo de la Red Humanitaria Nacional para formular e implementar proyectos de apoyo humanitario a las poblaciones más afectadas tanto de las ciudades como del campo. Algunos de los problemas detectados en las zonas afectadas donde se procedía a la respuesta humanitaria fueron la falta de atención de damnificados no ubicados en albergues y el limitado apoyo a los productores rurales y comerciantes que fueron afectados por la destrucción de la infraestructura de riego y de cultivos, así como por la destrucción de caminos y carreteras.

En agosto del año 2017 el Gobierno dio inicio a la"Reconstrucción con cambios" que debería permitir en un plazo de tres años invertir 25 mil millones de soles para la recuperación de los daños causados por las inundaciones y a la vez estimular el crecimiento económico en el país. Para ello la autoridad de la reconstrucción elaboró el plan correspondiente que debería asignar recursos a los sectores o ministerios para que estos contraten las obras que el sector privado ejecutaría. Los gobiernos locales y regionales podrían presentar sus propuestas y proyectos y, eventualmente, hasta ejecutar algunos siempre que el sector correspondiente le asignara los recursos del caso. Un criterio para la entrega de fondos a los municipios era que demuestren un $65 \%$ de ejecución presupuestal efectiva. Se consideró la opción de "obras por impuestos" como una alternativa para atraer la iniciativa privada (Soluciones Prácticas, 2017). 
La "Reconstrucción con cambios" que fue originalmente prevista para tres años ha tenido diversas complicaciones y conflictos entre las distintas instancias ejecutoras, lo que devino en la renuncia de dos de sus jefes y la iniciativa de hacer uso de convenios con algunos gobiernos para poder ejecutar más rápidamente las obras previstas. Sin embargo algunos proyectos claves orientados a la reducción de riesgos en las cuencas no serán ejecutados con los presupuestos existentes.

A diferencia de la respuestas anteriores ante las situaciones de emergencia presentadas por las bajas temperaturas, el Gobierno diseño e implementó un plan multianual y multisectorial ante las heladas y el friaje. Ello permitiría mejorar la atención de las emergencias e incorporar en las zonas alto-andinas y en la Amazonía acciones de reducción de la vulnerabilidad para lograr mejores condiciones térmicas en las viviendas campesinas. Esta iniciativa representa un avance pues se están incorporando medidas de reducción de riesgo y atención de emergencia en zonas donde la atención ha sido siempre muy limitada: Sin embargo es necesario evaluar hasta que punto y como se logrará atender tales necesidades para la mayor parte de la población vulnerable.

El 15 de marzo, el gobierno declaró la emergencia sanitaria ante el Covid 19, dada la amenaza de carácter global y la preocupación por la limitada capacidad de respuesta en el país.

Durante los meses siguientes el número de casos positivos comenzó a crecer rápidamente dentro y fuera de Lima por lo que se prolongaron las medidas iniciales por varios meses para flexibilizarlas progresivamente.

Las medidas tuvieron un impacto negativo en el empleo y las economías familiares, lo que se ha tratado de mitigar mediante la asignación de bonos de ayuda a la población en situación de pobreza y pobreza extrema.

Lo que viene ocurriendo en el Perú tiene que ver con vulnerabilidad institucional manifiesta en la precariedad política e institucional agravada por la corrupción; el abandono por décadas del sistema público de salud; la vulnerabilidad económica de la población y la carencia de una estrategia de gestión integral del riesgo que incorpore la salud, la economía y las estrategias de organización e inclusión social.

\section{Reflexiones finales}

- Los desastres y epidemias han sido relegados de la memoria histórica oficial y académica implicando aprendizajes limitados y la reproducción e incluso amplificación de los riesgos que los generan. 
- Las causas de los desastres han tendido erróneamente a ser definidas mediante el daño probable causado por un fenómeno de origen natural, soslayando la vulnerabilidad, las relaciones sociales y las capacidades de las organizaciones. La posibilidad de conocer mejor los escenarios futuros depende más de la comprensión de los procesos que generan los riesgos, que de la fenomenología.

- Las dinámicas económicas y sociales van determinando condiciones de riesgo mediante la ocupación del territorio, el incremento y concentración de la población, el deterioro de los ecosistemas, el limitado acceso a la vivienda y medios de vida seguros, y respuestas diferentes frente a los desastres. En contraste existen también prácticas que contribuyen a la reducción de los riesgos y los efectos de los desastres como la organización y solidaridad social, la reforestación, y el uso de tecnologías y conocimientos ancestrales que permiten reducir los peligros.

- La pobreza incide en las condiciones de riesgo dado el acceso limitado a condiciones de vida y servicios públicos seguros, y dada la carencia de recursos materiales para recuperarse de los desastres. El mayor o menor impacto de los desastres no sólo han sido las víctimas directas de éstos, sino sus efectos en la economía y en el incremento de la pobreza de la población afectada .

- La preocupación por el impacto de los fenómenos destructivos ha estado asociada a los riesgos en las actividades económicas hegemónicas, como fue el salitre, el guano, la caña de azúcar y la pesca. La atención a las personas afectadas y la recuperación hasta los años 1940 ha dependido de los recursos y capacidades locales ante la debilidad del Estado.

- A partir de la década de 1940 se producen migraciones hacia las ciudades, que en algunos casos están influidas por el impacto de los desastres desencadenados por la sequía, los aluviones y los sismos.

- El Gobierno Central asume la atención de las emergencias en las ciudades y los procesos de reconstrucción con estrategias diferentes que posibilitan la reducción parcial de los riesgos pero con recurrentes conflictos de competencia con las instancias municipales y con denuncias de corrupción. Algunos desastres frecuentes en las comunidades alto-andinas son ignorados por el Estado hasta fines de la década de 1,990, como fue el caso de los desencadenados por las heladas en las zonas alto-andinas y los friajes en la Amazonía.

- Los desastres de mayor impacto han incidido en cambios sociales, políticos, económicos, culturales e institucionales significativos en el país; entre estos últimos el de 1970 que dio origen al Sistema Nacional de Defensa Civil y el de 2007 que influyó en la creación del Sistema Nacional de Gestión de Riesgos. 
- La formación del Sistema Nacional de Defensa Civil fue posible en un contexto de reformas, proteccionismo, nacionalismo y participación de la población; en contraste con la formación del SINAGERD en un contexto de hegemonía del modelo neoliberal, crecimiento económico y de exacerbación de lo individual.

- Los desastres a partir de la década de 1990, evidenciaron la insuficiente delimitación de competencias entre las instituciones gubernamentales; el debilitamiento de las organizaciones de la población; y un aumento de la solidaridad pública inducida por los medios de comunicación. La atención a las emergencias y la reconstrucción estatal en las ciudades tuvo que afrontar mayores retos debido a la carencia del saneamiento físico y legal de las viviendas afectadas o destruidas; en contraste las ONG desarrollaron programas en las zonas rurales y periféricas de las ciudades en los que se implicó a la población afectada y a algunos gobiernos locales.

- Los cambios operados bajo la influencia de las Estrategias Internacionales permitieron incorporar enfoques de derechos e inclusión; los cambios en la tecnología, la infraestructura y los servicios públicos posibilitó mejoras sustantivas en la respuesta a emergencias.

- La reconstrucción ha estado más centrada en la infraestructura estatal y en créditos poco accesibles para los pobres; sólo en las últimas décadas tiene en cuenta la recuperación de la población más afectada. En muchas comunidades rurales la reconstrucción es fundamentalmente autoconstrucción. La reconstrucción después de cada desastre suele ser inconclusa.

- La Gestión del Riesgo de Desastres (GRD) surgió en América Latina como una perspectiva social crítica a la gestión gubernamental que se centraba en la respuesta de las instituciones especializadas en emergencias; la GRD propugna la participación de los actores del desarrollo, y en particular la comunidad organizada.

- La GRD gubernamental actualmente se ha centrado en los fenómenos naturales y en la vulnerabilidad de las nuevas edificaciones; ha incorporado la tecnología de la información para geo-referenciar la exposición a fenómenos peligrosos y los desastres y ha mejorado la capacidad de respuesta desde las instancias centrales de gobierno. Sin embargo, no ha logrado un fortalecimiento significativo de las capacidades de las instituciones regionales y locales y de la población.

- Las políticas de GRD no han tenido suficientemente en cuenta la investigación sobre la dimensión social de la vulnerabilidad, el incremento de la vulnerabilidad como consecuencia de la informalidad de las viviendas y el empleo; el 
deterioro de las construcciones muy antiguas, la tugurización y la vulnerabilidad extrema de las comunidades rurales ante las sequías.

- La educación formal y comunitaria debe ser priorizada en la GRD. Si bien existen algunos avances, la GRD no ha sido incorporada en la currícula de la mayoría de las universidades ni de los institutos de formación tecnológica. La educación comunitaria para la GRD así como las estrategias de comunicación constituyen temas pendientes.

\section{Bibliografía}

ALVA, J. (1983). Breve historia del fenómeno de la licuación de suelos en el Perú.

Banco Central de Reserva del Perú $(1925,1957)$. Memorias. Lima.

BARREAU, L. y PEÑA, P. (s/f). Gestión de crisis en terremotos pasados en el Perú. Lima: COOPI.

BARRIGA, V. (1951). Terremotos de Arequipa. Arequipa: Editorial La Colmena.

BECK, U. "La sociedad del riesgo global”. Siglo XXI Editores.

BONILLA, H. (1980). Un siglo a la deriva. Ensayos sobre el Perú, Bolivia y la guerra. Lima: IEP.

Comisión de la Verdad (2003)

CUETO, M. (1997). El regreso de las epidemias. Salud y sociedad en el Perú del siglo XX, Lima: Instituto de Estudios Peruanos, p. 256.

Chuquisengo, O. (2005). El fenómeno El Niño en Perú y Bolivia. Lima: ITDG, p. 197.

EGUIGUREN, V. (1895). Las Iluvias en Piura: fundación y traslaciones de la ciudad de San Miguel de Piura. Lima: Imprenta Liberal.

El Comercio, 2 enero 1878; 2 de Marzo 1,878; 3 octubre 2019.

FeRnÁNDEZ, M. (2007). Arica 1868 un tsunami y un terremoto. Archivos y Museos. Santiago: Dirección de Bibliotecas, p. 338.

FEYJOO DE SOUZA, M. (1984). Relación descriptiva de la ciudad y provincia de Trujillo del Perú. 2 volúmenes (1763). Lima: Banco Industrial del Perú.

FERRADAS, P. (2000). Las aguas del cielo y de la tierra. Impacto del Fenómeno El Niño en el Perú: Enfoques y experiencias locales. Lima: Centro de Estudios y Prevención de Desastres (PREDES) y Acción Ecuménica Sueca DIAKONIA.

FERRADAS, P. (2012). Riesgo de desastres y desarrollo. Lima: Soluciones Prácticas, p. 218.

FERRADAS, P. (2015). La memoria es también porvenir. Historia mundial de los desastres. Lima:

Soluciones Prácticas.

FLORES, A. (1997). Arequipa y el sur andino: ensayo de historia regional (siglos XVII-XX). Lima: Editorial Horizonte.

Franco, E. (1998). El Niño en el Perú: viejos y nuevos temas. En Desastres y Sociedad No. 9, Año 6, Enero-Diciembre. Lima 
GARCíA, V. (1996). Historia de los desastres en América Latina. México D.F.: La Red- Ciesas.

GIESECKE, A., SILGADO, E. (1981). Terremotos en el Perú. Lima: Ediciones Rikchay Perú.

GOOTEnBERG, P. (s/f). Población y etnicidad en el Perú republicano (siglo XIX) Algunas revisiones.

Documento de Trabajo № 71, Lima, IEP.

HumboldT, A. (1874). Cosmos; ensayo de una descripción física del mundo. Madrid: Imprenta de Gaspar y Roig.

IGP (2013). boletín152

INDECI (2019). Lecciones aprendidas terremoto de Pisco del 2007. Lima: Indeci.

KLEIN, N. (2010). "La doctrina del Shock” En El auge del capitalismo del desastre. Paidós Ediciones, p. 701.

PINTO, J. La construcción social del estado en el Perú: El régimen de Castilla y el mundo popular, 1845-1856.

PNUD (2020). Vulnerabilidades: más allá de la pobreza. Lima. p. 46.

KUONG, L. (1982). Retazos de la Historia de Moquegua.

Lossio, J. y BARRIGA, E. (Editores) (2017). Salud pública en el Perú del siglo XX: Paradigmas, discursos y políticas. Lima: Pontificia Universidad Católica del Perú.

MARIÁTEGUI, J. (1994). Mariátegui total, tomo I, Lima.

MIDDENDORF, E. (1973). Observaciones y estudios del país y sus habitantes durante una permanencia de 25 años. 3 tomos. Lima: Universidad Nacional Mayor de San Marcos. Perú.

MINISTERIO DEL AMBIENTE (s/f). Lo que el agua se llevó. Lima.

Ministerio del AmbIente (2016). Historia ambiental del Perú Siglo XVIII y XIX. Lima, 464 pp.

MONTORO, B. y FERRADAS, P. (2005). Reconstrucción y gestión de riesgo: Una propuesta técnica y metodológica. Lima: Soluciones Prácticas - ITDG.

PERALTA CASANI (2017). Los efectos de los desastres naturales en la producción vitivinícola de Moquegua y Arequipa (1600-1868). Arequipa: Universidad San Agustín.

PÉREZ, P. (1982). La población de Lima en el siglo XVIII. Universidad Autónoma de Madrid.

PrIETO, M. El Niño 1877/78. en Sudamérica. Un estudio de caso. IANIGLA-CCT-CONICET.

Polo, J. (1913). Apuntes sobre las epidemias del Perú. En Revista Histórica, Tomo V, Lima.

RAIMONDI, A. (1873). El departamento de Ancash y sus riquezas minerales. Lima: Imprenta de "El Nacional".

RED DE Estudios Sociales (1994). Desastres y Sociedad. Especial: Desbordes, Inundaciones y Diluvios. Agosto-Diciembre 1994 / No.3 / Año 2

REMY, M. (2013). Historia de las comunidades indígenas y campesinas del Perú. Lima: IEP. Revista Latinoamericana de Comunicación Chasqui (1991). Quito, FLACSO.

Rosales, S. y Vallejo, R. (2015). El eterno retorno del fenómeno de las heladas en el Perú: ¿Existen adecuadas políticas para combatir dicho fenómeno en el Perú? Lima: Universidad San Martín. 
RUIZ, I. y CASTRO, R. (2000). Los efectos del terremoto de 1868 en lquique y la provincia de Tarapacá: Opinión pública, vulnerabilidad urbana, fenómenos naturales y desastre en un escenario de crisis económica y política. (Perú, segunda mitad del siglo XIX). Santiago, p. 38.

RocHA, A. (2007). Los aluviones de 1981 y la iniciación de las investigaciones sobre el Fenómeno El Niño (ENSO) en el Perú y El Meganiño 1982-83. Lima.

ROMERO, G. (1985). Huaicos e inundaciones en el valle del Rímac. Lima: CIED y PREDES, p. 56.

SAN BARTOLOMÉ, RIVERA, DURÁN, MuÑoz y QUIUn (2015). Estudio experimental de una técnica de reforzamiento para edificaciones existentes con problemas de columna corta. Lima: Proyecto SENCICO-PUCP.

SEINER, L. (2002). Estudios de historia medioambiental. Perú, siglos XVI-XX. Lima: Universidad de Lima, Edición impresa.

SEINER, L. (S/F). Historia de los sismos en el Perú. Catálogo Siglo XVIII y XIX.

SEINER, L. (2001). El fenómeno El Niño en el Perú: Reflexiones desde la historia. Debate Agrario № 33. Lima, CEPES.

SENAMHI (2014). El fenómeno El Niño. Lima.

SOlUCIONES PRÁCticas (2017). Perú 2017: Riesgos, desastres y reconstrucción. Lima, p. 14.

TAMAYO, L. (1978). Historia social del Cuzco republicano. Lima, p. 335.

TAKAHASHI, K. (2014). Variedades de El Niño. Boletín técnico. Generación de modelos climáticos para el pronóstico de la ocurrencia del fenómeno EI Niño. IGP.

TAKAHASHI, K. (2016). The very strong EI Niño in 1925 in the far-eastern Pacific, climate Synamics. UNANUE, H. (1940). Observaciones sobre el clima de Lima 1806. Cuarta edición. Lima.

YEPES, E. (1980). Los inicios de la expansión mercantil capitalista en el Perú (1890-1930). En Historía del Perú Contemporáneo. Tomo VIII. Lima, Editorial Mejía Baca, p. 411.

VACCINE (2011). The 1918-1920 influenza pandemic in Peru. Junio.

VALCÁRCEL, C. (1915). El proceso de Putumayo. Lima: Imp. Comercial, p. 410.

VALCÁRCEL, L. (1981). Memorias. Lima: IEP, p. 499.

VALDIVIA, M. (2017). Acciones estatales antes y durante la epidemia de cólera de 1991.

VISCHER, A. (2008). Reconstrucción con prevención. Qué hacer, qué no hacer en la reconstrucción de viviendas luego de un sismo. Lima: Soluciones Prácticas - ITDG; p. 29.

WITT, H. (1992). Diario 1824-1890: un testimonio personal sobre el Perú del siglo XIX. Vol. (1824-1842). Lima: Banco Mercantil.

Woodman, R. (1985) “Recurrencia del fenómeno 'El Niño' con intensidad comparable a la del Niño 1982-1983". Ciencia, tecnología y agresión ambiental: El fenómeno El Niño. Lima: Concytec. 